Research Article

\title{
Green Technology Innovation Behavior of Cable Enterprises Based on Fuzzy Analytic Hierarchy Process of Internet of Things
}

\author{
Xiaowei Xu $\mathbb{D}^{1,2}$ Liangqun Qi, ${ }^{1}$ and Jianyu Sun ${ }^{2}$ \\ ${ }^{1}$ College of Economic and Management, Harbin University of Science and Technology, Harbin 150080, Heilongjiang, China \\ ${ }^{2}$ Zhongli Group, Changshu 215500, Jiangsu, China \\ Correspondence should be addressed to Xiaowei Xu; xxw83@hrbust.edu.cn
}

Received 20 August 2021; Revised 28 September 2021; Accepted 21 October 2021; Published 22 November 2021

Academic Editor: Sang-Bing Tsai

Copyright (c) 2021 Xiaowei Xu et al. This is an open access article distributed under the Creative Commons Attribution License, which permits unrestricted use, distribution, and reproduction in any medium, provided the original work is properly cited.

With the rise of global sustainable development, green technology innovation has also become the focus of academic research. This article aims to conduct research based on the abstract concept of the Internet of Things behavior based on green technology. A matrix is constructed based on the collected sample data to calculate and analyze so as to intuitively grasp the current situation of the entire industry. From the above data calculated according to the fuzzy hierarchy comprehensive evaluation method, it can be seen that the comprehensive score of the green technology innovation behavior of cable companies is 64.261 points, which belong to the left end of the range between 60 and 80 in the rating scale; in other words, the company's innovation capability has a certain degree of stability. The degree has just reached a "high" level, and there is still a lot of room for improvement. The efficiency weight (0.3343) score of the enterprise's benefit distribution mechanism is relatively low at 55.919. For managers of cable companies, green innovation is fundamental, and the awareness of green innovation should be improved to further promote the development of green technology innovation for the entire cable company.

\section{Introduction}

In recent years, green and low-carbon have become the main trends in the development of global technological innovation. Contemporary cable companies also focus on green technology innovation and regard it as one of the research priorities in the field of technology innovation for cable companies. Compared with traditional technological innovation, green technological innovation has greater ecological and economic value. It is the product of both social and economic development and environmental protection from pollution. How to meet the green consciousness innovation in the industrial process of raw materials, production, transportation, storage, and reuse of waste materials has become a major challenge in the operation of contemporary cable companies.

1.1. Meaning. Compared with traditional technological innovation, green technological innovation has greater ecological and economic value. In general, management innovation and technological innovation for environmental protection purposes are collectively referred to as green technological innovation. The unprecedented green technology innovation is linked with the fuzzy analytic hierarchy process, and a platform is built at the level of the Internet of Things, which meets the diversified standards.

1.2. Following Preparing Works. This research aims to promote and enrich the scientific debate about the phenomenon known as the Internet of Things (IoT) from a management perspective. Through the perspective of management and innovation literature, Caputo A studied the main facts of the Internet of Things and developed a conceptual framework to explain its development. The framework is then applied to the case of three-dimensional (3D) printing technology for additive manufacturing. However, the relevant aspects of green technology innovation are not reflected in the research [1]. Fuzzy analytic hierarchy process (FAHP) and analytic hierarchy process (AHP) of calculation process are a systematic analysis 
method that combines qualitative and quantitative. This method provides a basis for quantitative evaluation indicators for selecting the best design and has been widely used. ZhiQiang Ma mentioned that in the fuzzy analytic hierarchy process, when making similar comparisons between factors, because uncertain linguistic variables cannot be converted into definite values, the concept of triangular fuzzy numbers should also be introduced. However, due to the complexity of the algorithm for constructing fuzzy AHP, it has not been popularized in many fields [2]. In studying the basic idea of fuzzy analytic hierarchy process, Ehsan $\mathrm{N}$ established a hierarchy structure from bottom to top. He proposed that when making a decision, first a multilevel hierarchical model is complied for each element of the decision-making problem, then a fuzzy judgment table is created, then the relative importance of each element is calculated, and finally a scientific basis is provided for decision-makers to choose. However, the accuracy of the data sources in the research will greatly affect the final scoring and analysis results, making the screening of statistical data occupy most of the research time and energy [3].

1.3. Innovation in Research. The innovations of this research are mainly reflected in the following aspects:

(1) On the basis of literature research, the definition of "green technological innovation" has been improved, and the definition, concept, and connotation of green technological innovation have been refined by taking the characteristic socio-economic development and environmental protection as the starting point. On this basis, a hierarchical model is built based on the Internet of Things.

(2) The fuzzy analytic hierarchy process is used to solve the problem that the concept of innovative behavior research is difficult to directly measure. Constructing abstract problems with algorithm models is convenient for intuitive grasp.

\section{Green Technology Innovation Behavior of Cable Companies Based on the Fuzzy Analytic Hierarchy Process of the Internet of Things}

2.1. IoT System Architecture. The structure of the Internet of Things system can be divided into the perception layer to obtain data, the network layer to transmit information and preprocessing, and the application layer provides services to users, a total of three layers. The realization of the tasks and services of the corresponding layers needs to be supported by the relevant technologies of each layer. The relevant architecture diagram is shown in Figure 1 [4].

Among the Internet of Things technology, RFID technology and ZigBee technology are important components $[5,6]$, and they have been widely used and studied in various fields. Compared with traditional tags, Table 1 shows the technical advantages of RFID over traditional tags.

In recent years, as our country attaches great importance to the Internet of Things, RFID technology is the cornerstone of the Internet of Things, and its various technologies have made rapid progress in our country and have begun to be applied in many fields. With the rise of the application of the Internet of Things in contemporary society, RFID technology, as an important part of it, will continue to maintain a trend of rapid development in the future social development, and the areas covered are increasing day by day [7].

In the ZigBee network, ZigBee nodes need to complete specific network function missions and corresponding parameter settings [8]. According to the functions that the ZigBee node needs to realize, it can be divided into three parts: router, coordinator, and terminal node [9].

2.1.1. Router. After the router joins the ZigBee network, the router mainly completes the task of data transmission in the ZigBee network. Under normal circumstances, in order to ensure the stability and reliability of the ZigBee network, the router must be in working mode, so the power consumption of the router requires more in-depth research [10].

2.1.2. Coordinator. The function of the coordinator is relatively complete, which can realize the connection control of the overall network. After selecting the appropriate channel and network symbol, it can complete the startup and create the network. The coordinator plays the role of a router after the basic work such as creating a network is completed [11] and performs functional tasks such as information transmission and storage $[12,13]$.

2.1.3. Terminal Node. The terminal node is used to realize the collection of external environment or human body sign information. In order to reduce the energy consumption of the terminal node, it can usually be set to a sleep mode or a wake-up state. One of the topological structures can be selected in the overall network construction, or they can be combined to form a new topological structure according to the advantages of each topological structure [14].

The topological structure used by the system is different, the corresponding model is different, and the parameter settings of each node in the network are also different. Table 2 compares the characteristics of three different ZigBee topologies [15, 16].

2.2. Analytic Hierarchy Process. The analytic hierarchy process has the characteristics of simple calculation $[17,18]$. The shortcomings of the general process of the analysis level are solved; that is, the consistency of the standard table cannot meet the requirements, and the scale is relatively cumbersome $[19,20]$. Introducing the fuzzy analytic hierarchy process into the business stability measurement model makes the stability selection measurement process clearer and the calculation process easier [21, 22], thus providing a simple and effective method for the measurement of enterprise stability [23, 24]. Figure 2 shows the decision structure diagram of the analytic hierarchy process [25]. 


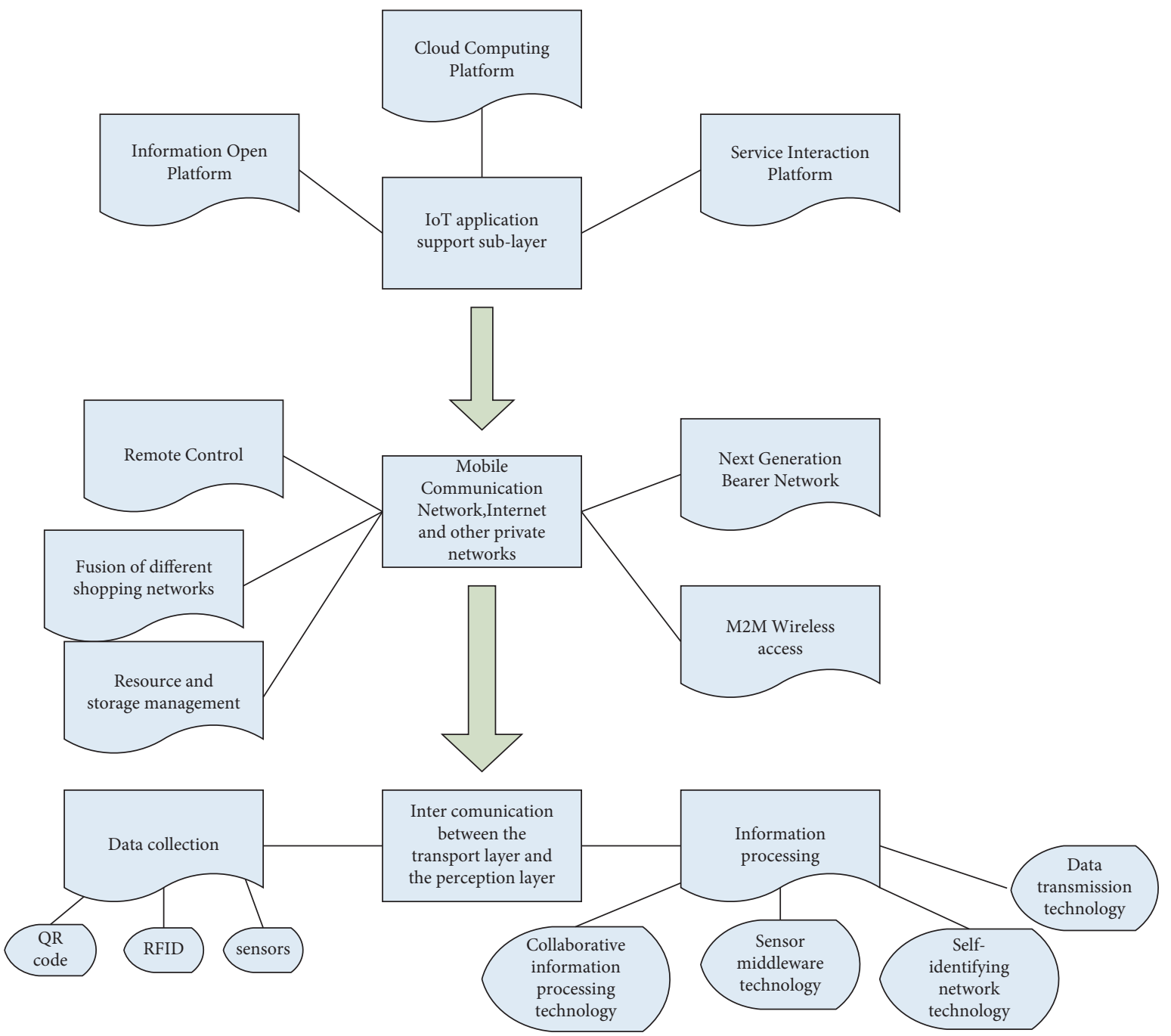

FIGURE 1: Internet of Things technology architecture chart.

TABLE1: Traditional tag and RFID tag features contrast.

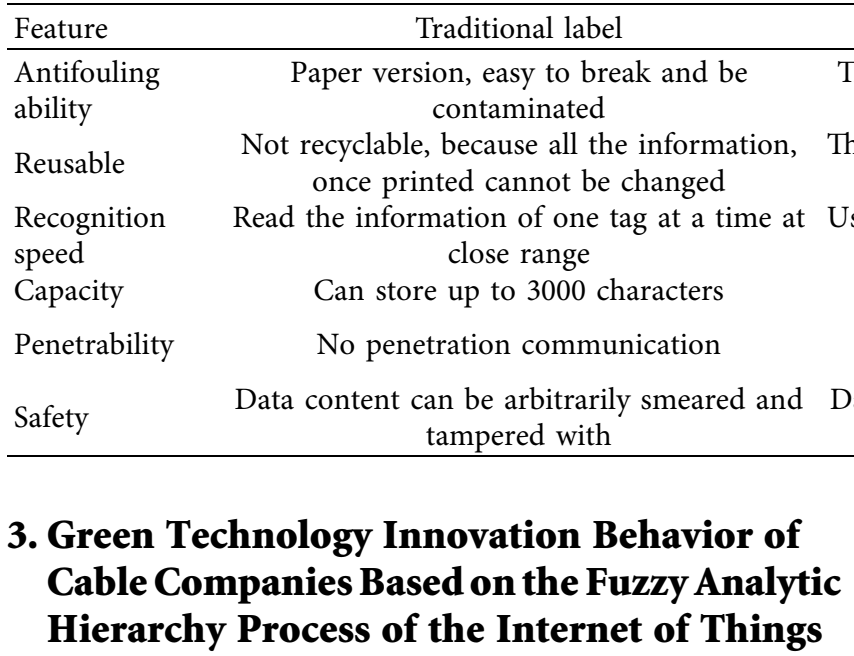

3.1. Build a Hierarchical Model. Figure 3 shows a more common three-level (multilevel can be analogized) structure model.

\section{RFID label}

The label uses a chip to store information, so it is resistant to strong pollution capacity

added, or deleted and other operations, so it can be recycled

ectromagnetic coupling, it can be long-distance read multiple tags at once

The amount of stored data is dozens of times that of barcodes

Can penetrate wood, metal, plastic, and so on. The advantage of nontransparent material is it can penetrate communication content realizes password through encoding and protection, and internal information is not easy to be forged and changed
This is a grouping and stratification of multiple evaluation indicators according to the nature of the problem and according to the top level (objective a), the middle level (first-level evaluation index $b_{\mathrm{i}}, i=1,2, \ldots, m$ ), and the bottom level (two A three-level (or multilevel) evaluation index system arranged in the form of $C_{i j}, i=1,2, \ldots, m ; j=1,2, \ldots$, $\left.n_{i}\right)$. Then, we build the fuzzy judgment matrix. 
TABLE 2: Characteristics of ZigBee network topology.

\begin{tabular}{ll}
\hline Type & Typical characteristics \\
\hline $\begin{array}{l}\text { Star topology } \\
\text { performance, but there is only one data transmission path, and the coordinator may affect the transmission of network } \\
\text { data, which can be used in data transmission small scene }\end{array}$ \\
$\begin{array}{l}\text { Cluster } \\
\text { topology }\end{array}$ \\
The structure is complex, and the network can be transmitted in a multihop form; it has the performance characteristics of \\
self-organization and self-coordination
\end{tabular}

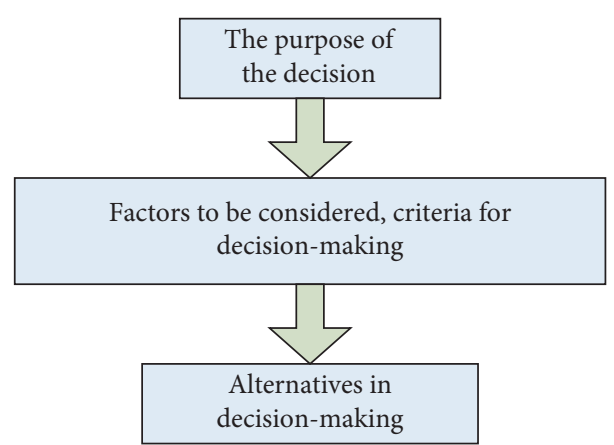

FiguRE 2: Decision structure diagram of analytic hierarchy process.

To construct the fuzzy evaluation matrix, first, the single factor in the hierarchical structure model in Figure 3 needs to be considered. $\operatorname{Cij}\left(i=1,2, \ldots, m ; j=1,2, \ldots, n_{i}\right)$ is a single factor evaluation. From the factor $b_{i}(i=1,2, \ldots, m)$, focusing among the elements of the belonging relationship, $C_{i j}$ has the membership degree of $a_{j j}$ to the judgment set $v_{j}(j=1,2, \ldots$, $\mathrm{n})$, so that the single factor judgment set of the $j$-th element $C_{i j}$ under the $b_{i}$ grouping is obtained:

$$
d_{m}=\left(d_{n 1}, d_{n 2}, \ldots, d_{n n}\right)
$$

In this way, the evaluation set of nj factors constructs an evaluation matrix $D_{m}(m=1,2, \ldots, i)$; that is, each evaluated object is determined from $b_{i}(i=1,2, \ldots, m)$. The fuzzy relationship to $C$, that is, the matrix is as follows:

$$
D_{m}=\left(a_{j j}\right)_{i_{m} \times i}=\left[\begin{array}{cccc}
a 11 & a 12 & \ldots & a 1_{i} \\
a 21 & a 22 & \ldots & a 2_{i} \\
\ldots & \ldots & \ldots & \ldots \\
a_{i_{n} 1} & a_{i_{n}} & \ldots & a_{i_{n} i}
\end{array}\right] .
$$

Specifically, $a_{j j}$ 'represents the frequency distribution of the $i$ factor $C_{i j}$ on the $i$ th comment $v_{j}$ ' under the $b_{i}$ grouping.

\subsection{Determining the Weight of the Green Technology Inno-} vation Stability Measurement Index of Cable Companies. The index weight of the green technology innovation stability measurement index of the cable enterprise will be determined by the fuzzy analytic hierarchy process, taking into account the application level.
This article will use the priority relationship matrix and the fuzzy judgment matrix as the basis to introduce the fuzzy consensus judgment that is inherently consistent.

Specific steps are as follows.

First, the optimal matrix $D$ needs to be constructed:

$$
D=\left(d_{i j}\right)_{n i \times n i}
$$

The optimal matrix $D$ is defined as a three-valued matrix $(0,0.25,0.5)$ on a finite universe $C=(c 1, c 2, \ldots, c n)$. Obviously, $D$ is a fuzzy complementary matrix.

Second, the fuzzy consistent judgment matrix $G$ is constructed:

$$
G=\left(g_{i j}\right)_{n i \times n i}
$$

After the optimization table is established, it is converted into a fuzzy consistency matrix. The fuzzy consistent judgment matrix $G$ is a multivalued matrix $G=\left(g_{i j}\right)_{n i \times n i}$ on the finite universe $A=\left(a 1, a 2, \ldots, a_{n}\right)$ and $0<g_{i j}<1 . G_{i j}$ shows the relative importance of $a_{i}$ to $a_{j}$ : if $0<g_{i j}<0.25$, it means that aj is more important than ai, and the smaller the $g_{i j}$ is, the more important $a_{j}$ is than $a_{i}$; if $g_{i j}=0.25$, it means that ai and aj are equally important; if $0.25<g_{i j}<0.5$, it means that ai is more important than aj, and the larger the $g_{i j}$, the more important it is.

In the conversion process, the preferred matrix $D=\left(d_{i j}\right)_{n i \times n i}$ needs to be summed by rows, namely:

$$
T_{j}=\sum_{k=1}^{n_{j}} e_{j k}, \quad j=1,2, \ldots, n_{j} .
$$

Then, 


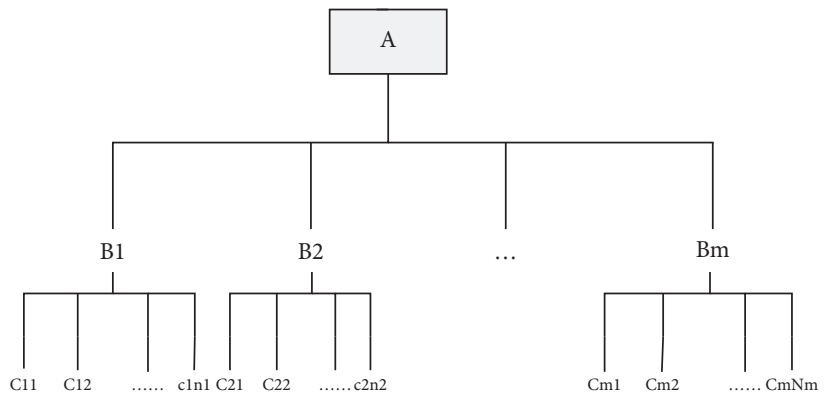

FIgURE 3: The hierarchical model of problem evaluation.

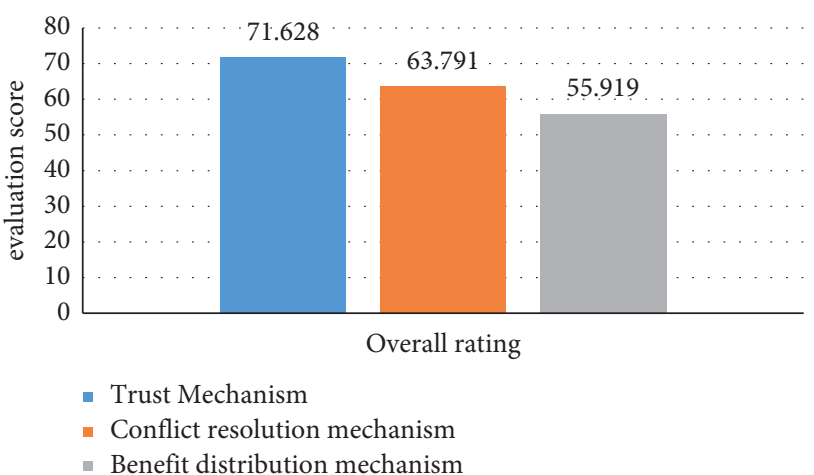

FIGURE 4: The comprehensive scoring table for green tech innovation behavior of cable companies.

$$
g_{i j}=\frac{t_{i}-t_{j}}{2 n_{j}}+0.5 \text {. }
$$

The final matrix $G=\left(g_{i j}\right)_{n i \times n i}$ is the fuzzy consistency matrix.

Then, the matrix's $G=\left(g_{i j}\right)_{n i \times n i}$ max is calculated. The normalized feature vector is the standard weight set $w i^{T}$.

3.3. Layered Comprehensive Evaluation. After determining the index weights of each layer in the evaluation system, the lower-level indicators need to be adjusted according to the characteristics of the constructed index system.

According to the fuzzy judgment matrix $D_{m}$ established above and the calculated weight set $w_{i}^{T}$, the evaluation vector $B_{j}$ of the second layer is calculated:

$$
B_{j}=w_{i}^{T} \times D_{m}, \quad(i=1,2, \ldots, m) .
$$

3.4. High-Level Comprehensive Evaluation. Using the results of the hierarchical comprehensive evaluation, the comprehensive evaluation matrix $D$ can be obtained. Based on this, the comprehensive evaluation can be obtained. Valence vector is as follows:

$$
C=w^{t} \times D
$$

The hierarchical evaluation vector $B_{j}$ and the comprehensive evaluation vector $C$ are, respectively, multiplied by the comment score set $V$ to obtain the evaluation score of each index, and finally the evaluation result is analyzed.
3.5. Questionnaire Survey and Algorithm Construction. This article uses a questionnaire survey method to issue questionnaires to 15 experienced experts in the industry. The comprehensive evaluation matrix $B$ is obtained using the results of the hierarchical comprehensive evaluation, $W_{t}$ and $B$ are multiplied to obtain the comprehensive evaluation vector $\mathrm{C}$, and finally the hierarchical evaluation vector $B_{j}$ and the comprehensive evaluation vector $C$ are, respectively, multiplied by the comment score set $V$ to obtain the evaluation score $X_{i}(i=0,1,2,3)$ of each index as follows:

$$
\begin{aligned}
& X_{1}=C_{1} \times V^{t}=71.628, \\
& X_{2}=C_{2} \times V^{t}=63.791, \\
& X_{3}=C_{3} \times V^{t}=55.919, \\
& X=C \times V^{t}=64.261 .
\end{aligned}
$$

\section{Comprehensive Evaluation and Analysis}

4.1. Model Running Results. From the above data calculated based on the fuzzy hierarchy comprehensive evaluation method, it can be seen that the comprehensive score of the green technology innovation behavior of cable companies is 64.261 points, which belongs to the left end of the range between 60 and 80 in the rating scale, that is, the stability of the alliance has just reached a "high" level, and there is still a lot of room for improvement.

Among them, the effectiveness of the company's trust mechanism (weight: 0.4539 , the same below) scored 71.628, which is at a relatively high level. This shows that the sample 
company in the questionnaire survey strictly followed the contract, strictly protected the cooperation secrets $(0.2131)$, and accumulated a good social reputation (0.4539) for the company itself in the process, and also increased the number of alliance members. The interest dependence $(0.3343)$ has made a great contribution to the continuity and stability of the green technology innovation behavior of cable companies. The company's conflict resolution mechanism effectiveness (0.2131) score of 63.791 has just reached a high level. This is mainly due to the high degree of consistency (0.5000) of the strategies of experts and scholars in the division of labor at the level of green technology innovation. Finally, the company's benefit distribution mechanism effectiveness (0.3343) score of 55.919 is relatively low. Judging from the judgment matrix $\mathrm{X} 3$, it is mainly because the industry's collective benefit maximization (0.2131) awareness is generally weak and industry managers should pay attention to this point and propose solutions to improve the stability of industry practitioners, as shown in Figure 4.

\section{Conclusion}

This article focuses on the Internet of Things technology. Through the measurement of the sample company and sample data, it can be found that the company's overall stability score is 64.261 , indicating that the stability of the green and innovative industry constructed by the company has just reached a relatively high level, which is consistent with the reality, but there are still many problems. There is a lot of room for improvement, especially in the management and control of the effectiveness of the benefit distribution mechanism (55.919 points) In addition, the alliance's trust mechanism effectiveness score of 71.628 has reached a high level, while the conflict resolution mechanism's effectiveness score of 63.791 is not high, but it has reached a relatively satisfactory level, but there is still room for improvement. The results of the model operation also show that when alliance managers want to improve the stability of the alliance, they should focus on the design of the alliance's benefit distribution mechanism. The limitation of this article is that it is limited by the author's professional level and the small sample size. During the operation of the fuzzy system, there must be other important indicators that are not included in the model of this article. Therefore, this paper constructs this concept to make up for the shortcomings of the model. In future research, the sample size can be expanded, and more professional opinions and data samples of experts in green innovation technology can be referred to, and the research on green technology innovation behavior of cable companies can be analyzed from a more comprehensive perspective.

\section{Data Availability}

No data were used to support this study.

\section{Conflicts of Interest}

The authors declare that there are no conflicts of interest.

\section{Acknowledgments}

This work was supported by Jiangsu Postdoctoral Science Foundation in 2019.

\section{References}

[1] A. Caputo, G. Marzi, and M. M. Pellegrini, "The internet of things in manufacturing innovation processes:development and application of a conceptual framework," Business Process Management Journal, vol. 22, no. 2, pp. 383-402, 2016.

[2] Z. Q. Ma, "Research on the efficiency evaluation of emergency communication system via fuzzy analytic hierarchy process," Operations Research and Fuzziology, vol. 08, no. 4, pp. 173178, 2018.

[3] N. Ehsan, F. Dawud, and A. S. Mohsen, "Land suitability analysis for solar farms exploitation using GIS and fuzzy analytic hierarchy process(FAHP) - a case study of Iran," Energies, vol. 9, no. 8, pp. 643-647, 2016.

[4] S. Liu, "Research on the application of internet of things technology in digital sports and community fitness," Boletin Tecnico/Technical Bulletin, vol. 55, no. 4, pp. 139-145, 2017.

[5] T. Hu, J. Lv, and X. Qingsheng, "A novel human behaviour information coding method based on eye-tracking technology," Traitement du Signal;Signal-Image-Parole, vol. 34, no. 34, pp. 153-173, 2017.

[6] J. li and Y. B. Huang, "Guang chan, research and demonstration of traceability system of quinoa based on the technology of internet of things," Journal of Jilin Agricultural University, vol. 39, no. 5, pp. 624-630, 2017.

[7] J. Jiao, C. Zhou, and F. Feng, "Research on medlar circulation Internet of Things model based on RFID and WSN technologies," Wireless Internet Technology, vol. 15, no. 15, pp. 103-106, 2018.

[8] S. Li, L. D. Xu, and S. Zhao, "The internet of things: a survey," Information Systems Frontiers, vol. 17, no. 2, pp. 243-259, 2015.

[9] T. Yang, B. Yu, H. Wang, J. Li, and Z. Lv, "Cryptanalysis and improvement of Panda - public auditing for shared data in cloud and internet of things," Multimedia Tools and Applications, vol. 76, no. 19, pp. 19411-19428, 2017.

[10] V. Pande, C. Marlecha, and S. Kayte, "A review-fog computing and its role in the internet of things," International Journal of Engineering Research in Africa, vol. 6, no. 10, pp. 2248-96227, 2016.

[11] C. Perera, C. H. Liu, and S. Jayawardena, "The emerging internet of things marketplace from an industrial perspective: A survey," IEEE Transactions on Emerging Topics in Computing, vol. 3, no. 4, pp. 585-598, 2017.

[12] J. Liu and M. Zhao, "Does environmental regulation promote green technology innovation in Chinese manufacturing?" Open Access Library Journal, vol. 7, no. 7, pp. 1-16, 2020.

[13] F. L. Paula, F. C. Tiago, and S. A. Manuel, "A review on internet of things for defense and public safety," Sensors, vol. 16, no. 10, pp. 1644-1647, 2016.

[14] M. A. Razzaque, M. Milojevic-Jevric, and A. Palade, "Middleware for internet of things:A survey," IEEE Internet of Things Journal, vol. 3, no. 1, pp. 70-95, 2017.

[15] J. Lin, W. Yu, N. Zhang, X. Yang, H. Zhang, and W. Zhao, “A survey on internet of things: architecture, enabling technologies, security and privacy, and applications," IEEE Internet of Things Journal, vol. 4, no. 5, pp. 1125-1142, 2017. 
[16] O. Bello and S. Zeadally, "Intelligent device-to-device communication in the internet of things," IEEE Systems Journal, vol. 10, no. 3, pp. 1172-1182, 2016.

[17] J. Singh, T. Pasquier, and J. Bacon, "Twenty security considerations for cloud-supported internet of things," IEEE Internet of Things Journal, vol. 3, no. 3, pp. 269-284, 2017.

[18] X. Sun and N. Ansari, "EdgeIoT: mobile edge computing for the internet of things," IEEE Communications Magazine, vol. 54, no. 12, pp. 22-29, 2016.

[19] J. W. Xue, X. K. Xu, and F. Zhang, "Big data dynamic compressive sensing system architecture and optimization algorithm for internet of things," Discrete and Continuous Dynamical Systems - Series S, vol. 8, no. 6, pp. 1401-1414, 2017.

[20] Y. Yang, L. Wu, G. Yin, L. Li, and H. Zhao, "A survey on security and privacy issues in internet-of-things," IEEE Internet of Things Journal, vol. 4, no. 5, pp. 1250-1258, 2017.

[21] P. A. Laplante and N. Laplante, "The internet of things in healthcare: potential applications and challenges," It Professional, vol. 18, no. 3, pp. 2-4, 2016.

[22] I. Yaqoob, E. Ahmed, I. A. T. Hashem et al., "Internet of things architecture: recent advances, taxonomy, requirements, and open challenges," IEEE Wireless Communications, vol. 24, no. 3, pp. 10-16, 2017.

[23] A. Mosenia and N. K. Jha, "A comprehensive study of security of internet-of-things," IEEE Transactions on Emerging Topics in Computing, vol. 5, no. 4, pp. 586-602, 2017.

[24] H. S. Dhillon, H. Huang, and H. Viswanathan, "Wide-area wireless communication challenges for the internet of things," IEEE Communications Magazine, vol. 55, no. 2, pp. 168-174, 2017.

[25] N. Kshetri, "Can blockchain strengthen the internet of things?" It Professional, vol. 19, no. 4, pp. 68-72, 2017. 